\section{Bioavailability of Strontium Ions from Bioactive Glasses In Vivo: A Micro- PIXE Study of Trace Elements at the Bone Interface}

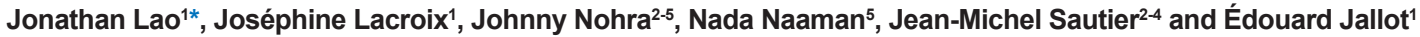 \\ ${ }^{1}$ Clermont Université, Université Blaise Pascal, CNRS/IN2P3, Laboratoire de Physique Corpusculaire, BP 80026, 63171 Aubière Cedex, France \\ 2INSERM, U872, Eq.5, Laboratoire de Physiopathologie Orale et Moléculaire, Paris, F- 75006, France \\ ${ }^{3}$ Centre de Recherche des Cordeliers, Université Pierre et Marie Curie, UMR S 872, Paris, F- 75006, France \\ ${ }^{4}$ Université Paris Diderot, Paris 7, UFR d'Odontologie, Paris, France \\ ${ }^{5}$ Department of Periodontology, Laboratory of Calcified Tissue, School of Medical Dentistry, Saint-Joseph University, Beirut, Lebanon
}

\begin{abstract}
Studying the local release of strontium traces in vivo is of key interest, but calls for highly sensitive techniques besides providing an excellent (micronic) spatial resolution. In this context nuclear microprobes such as the PIXE (Particle-Induced X-ray Emission) technique, appear as powerful tools of investigation. Here, the in vivo behaviour of a Sr-delivering bioactive glass has been investigated through micro-PIXE analyses in connection with histological studies. New bone formation is observed after 6 weeks of implantation in rabbit femoral condyle. Interestingly, Sr traces are detected over a large area at the site of implantation, demonstrating the efficient release of Sr osteo inductive ions from the glass and their diffusion over several ten microns through the tissues. From its inorganic composition and content in traces of interest such as Zn, neo formed bone seems of higher quality for Sr-delivering particles compared to Sr-free particles, evidencing the positive effect of $\mathrm{Sr}$ in vivo.
\end{abstract}

Keywords: Bioactive glasses; Strontium; Osteoinduction; Osteoproduction

\section{Introduction}

Recent advances in the field of biomaterials are the development of bioceramics releasing osteo inductive ions directly onto the site of implantation. Of special interest is the delivery of strontium since Sr has marked stimulatory effects onto bone cells resulting in strengthening of bone, stimulation of bone formation and decrease in bone resorption [1]. The benefits from the local administration of Sr from implants seem now well established in vivo, as increased osseo integration and bone apposition are reported for Sr-based implants [2-4]. But to date quantitative data are sparse about the amount of $\mathrm{Sr}$ ions really delivered onto the site of implantation. These data are however crucial for evaluating the right dose to deliver for positive effects, and matching the implant properties in view of this. Rare studies investigated the accumulation of $\mathrm{Sr}$ in bone, often in the case of oral administration of $\mathrm{Sr}[5,6]$. To our knowledge, the only measurement reported in the literature about the in vivo release of $\mathrm{Sr}$ from bioceramics is found in a study by Gorustovich et al. [7]. The authors indicated that $\mathrm{Sr}$ was not detected in neoformed bone after 30 days implantation, which is quite surprising given the relatively high amount (6 wt. \%) of SrO in the bioactive glass investigated. This could rather be due to the use of SEMEDXS: the limited sensitivity of electron X-ray microanalysis cannot allow the detection of $\mathrm{Sr}$ if only traces are present.

Obviously there is room for improvements in the collect of quantitative data on trace elements at the bone/implant interface. Here we wanted to demonstrate how useful can be a combined analysis coupling histological studies and $\mu$-PIXE together, with a special focus on the bioavailability of $\mathrm{Sr}$ ions from glasses in vivo, at a few ten p.p.m sensitivity.

\section{Materials and Methods}

$\mathrm{SiO}_{2}-\mathrm{CaO}$ (B75) and $\mathrm{SiO}_{2}-\mathrm{CaO}-\mathrm{SrO}$ (B75-Sr5) glasses were synthesized through the sol-gel- process, their respective composition being indicated in table 1. It is worth noting that the $\mathrm{SrO}$ content in B75-Sr5 is close to that in the study from Gorustovich et al. [7]. One adult male New Zealand White rabbit weighing $4 \mathrm{~kg}$ (aged 10 months)

\begin{tabular}{|c|c|c|c|}
\hline & $\mathrm{SiO}_{2}$ wt. $\%$ & CaO wt.\% & SrO wt.\% \\
\hline B75 & 75 & 25 & - \\
\hline B75-Sr5 & 75 & 20 & 5 \\
\hline
\end{tabular}

Table 1: Composition of implanted sol-gel glasses.

was used for the experiment. $2.5 \mathrm{~cm}$ long incision was made over the distal epiphysis of each femur in the medial aspect of the knee joint. Cylindrical bone defects were then created to a depth of $10 \mathrm{~mm}$ and filled with granules of either B75 or B75-Sr5. The rabbit was sacrificed at 6 weeks by intrapulmonary injection of $1.5 \mathrm{~mL}$ of embutramide/ mebenzonium iodide/tetracaine hydrochloride (Intervet). The specimen was immediately fixed in $10 \%$ neutral buffered formalin and embedded in resin following complete dehydration in ascending grades of ethanol. The undecalcified histological sections were then ground down to about $60 \mu \mathrm{m}$ and stained with Stevenel's Blue/Picrofuchsin. $\mu$-PIXE analyses were carried out on repolished histological sections to remove staining and were performed using a $3 \mathrm{MeV}$ proton beam of 1 $\mu \mathrm{m}$ diameter at the AIFIRA nanobeam line, CENBG, France.

\section{Results and Discussion}

Light microscopy of the core biopsy (Figure 1a and 1b) taken at the center of the defect demonstrated residual graft (shadow regions) surrounded by new bone. Whereas some particles were completely trapped in newly formed bone, others showed partial or no contact with woven bone. It appears that both glasses, i.e. B75 and B75-Sr5, were

*Corresponding author: Jonathan Lao, Clermont Université, Université Blaise Pascal, CNRS/IN2P3, Laboratoire de Physique Corpusculaire, BP 80026, 63171 Aubière Cedex, France, E-mail: lao@clermont.in2p3.fr

Received June 08, 2013; Accepted July 22, 2013; Published August 23, 2013

Citation: Lao J, Lacroix J, Nohra J, Naaman N, Sautier JM, et al. (2013) Bioavailability of Strontium lons from Bioactive Glasses In Vivo: A Micro-PIXE Study of Trace Elements at the Bone Interface. Bioceram Dev Appl S1: 004. doi: 10.4172/2090-5025.S1-004

Copyright: (c) 2013 Lao J, et al. This is an open-access article distributed unde the terms of the Creative Commons Attribution License, which permits unrestricted use, distribution, and reproduction in any medium, provided the original author and source are credited. 
Citation: Lao J, Lacroix J, Nohra J, Naaman N, Sautier JM, et al. (2013) Bioavailability of Strontium lons from Bioactive Glasses In Vivo: A Micro-PIXE Study of Trace Elements at the Bone Interface. Bioceram Dev Appl S1: 004. doi: 10.4172/2090-5025.S1-004

Page 2 of 3

osteoconductive and acted as a scaffold for osteogenic cell population and new bone in growth. Figure 1c- 1e also show the $\mu$ - PIXE chemical imaging of the elements and from the distributions it is visible that the so- called "glass" particles are in fact under mineralization, the original glass particles being at least partially changed into calcium phosphates containing small amounts of $\mathrm{Si}$ and Sr. Figure 2 exhibits the elemental concentration profiles obtained along the arrow indicated in Figure 1c, with increments of $25 \mu \mathrm{m}$ between each measure. Substantial modifications are observed along the bone/B75-Sr5 glass interface. The "glass" particles have endured partial dissolution, with only $10 \mathrm{wt}$ \% Si remaining, evidencing breakdown of the silicate network. The initially phosphorus-free glass particles have incorporated significant amount of P (ca. 12 wt.\%) after 6 weeks implantation, together with Ca (ca. 33 wt.\%) from the biological fluids. The content in trace elements also significantly differs from each side of the bone/glass interface, which is identified as the interface between zones $3 / 4$ in figure $2 \mathrm{Sr}$ efficiently diffused from the glass particles to the bone tissues in contact, with a decreasing $\mathrm{Sr}$ concentration from glass to bone: $4500 \mathrm{ppm} \mathrm{Sr}$ are found inside the glass particles at the bone interface (zone 4), $2910 \mathrm{ppm} \mathrm{Sr}$ in the tissues immediately in contact (zone 3), $844 \mathrm{ppm} \mathrm{Sr}$ at 25-50 $\mu \mathrm{m}$ away from the interface (zone 2), and $275 \mathrm{ppm} \mathrm{Sr}$ at $50-75 \mu \mathrm{m}$ from the interface (zone 1). These values are significant when compared to the initial $42300 \mathrm{ppm} \mathrm{Sr}$ (equivalent to $5 \mathrm{wt}$ \% $\% \mathrm{SrO}$ ) in the starting glass composition and to the Sr content in native bone tissues which is below
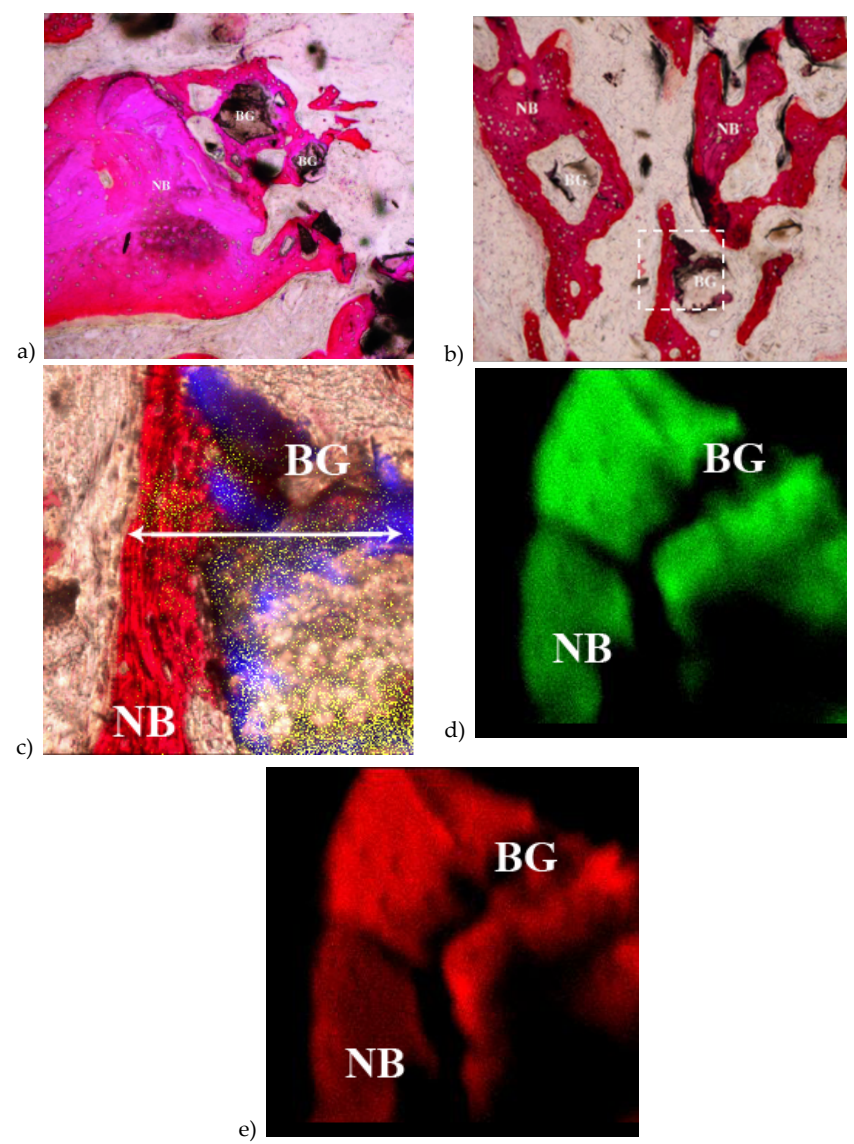

Figure 1: a), b) Optical views of stained histological sections showing B75$\mathrm{Sr} 5$ glass particles (BG) surrounded by new bone (NB) at the center of the defect, original magnification $\times 10$. c) Corresponding $\mathrm{Si}$ (blue) and $\mathrm{Sr}$ (yellow) distributions as obtained through PIXE imaging $\left(200 \times 200 \mu \mathrm{m}^{2}\right)$, superimposed to the initially stained section. Ca (green), $P$ (red) distributions are also shown independently in d) and e).
$100 \mathrm{ppm}$. The contents in other trace elements also differ when crossing the bone/glass interface: e.g. in the "glass" particles regions $\mathrm{Zn}$ and $\mathrm{Mg}$ decrease while $\mathrm{S}$ and $\mathrm{K}$ increase. From the histological stained sections as well as from the $\mu$-PIXE chemical imaging of the elements, three kinds of regions are identified: the "glass" particles under mineralization, the neoformed bone and the native bone tissues (out of the defect). Figure 3 shows the inorganic composition of the three regions depending on the type of glass: B75 (Sr-free) vs. B75-Sr5 (5 wt.\% SrO). The comparison is meaningful. From $\mathrm{Ca}$ and $\mathrm{P}$ concentrations, we observe the biomineralization process being more advanced inside B75-Sr5 particles. The $\mathrm{Ca} / \mathrm{P}$ atomic ratio is calculated as $1.65 \pm 0.24$ inside B75-Sr5 particles, close to the characteristic values of bone mineral, compared to $3.69 \pm 1.61$ inside B75. Although the starting B75-Sr5 composition owns 5wt.\% less Ca than B75 (Table 1), an average of 30.3 wt.\% Ca is detected inside the B75-Sr5 particles, compared to 19.6 wt. \% for $\mathrm{B} 75$ (data not shown). So the lower $\mathrm{Ca} / \mathrm{P}$ ratio for $\mathrm{B} 75-\mathrm{Sr} 5$ is due to a higher incorporation of both $\mathrm{Ca}$ and $\mathrm{P}$ ions from its environment. In the same manner the $\mathrm{Si}$ content is much lower inside B75-Sr5 particles after 6 weeks implantation: 10.5 wt. \% compared to 27.8 wt. \% inside B75. This may seem surprising since it has been previously shown that Sr-doped glasses have slower dissolution rates in physiological fluids [8], but these studies were lead in acellular media. From our point of view the quicker transformation of B75-Sr5 particles in vivo highlights the positive effect of $\mathrm{Sr}$ onto the mineralization process through osteogenic action [9]. In neoformed bone only small amounts of $\mathrm{Si}$ are detected for both types of glasses, demonstrating efficient elimination of the dissolution products out of the implantation site. The Si content is also lower for the tissues in contact with B75-Sr5 particles: $0.1 \mathrm{wt}$ $\%$ vs. 1.5 wt. \% for B75. The Sr content of course depends on the glass implanted. For B75, no difference in the Sr content is observed between native and neoformed bone (94 ppm Sr). For B75-Sr5 particles, Figure
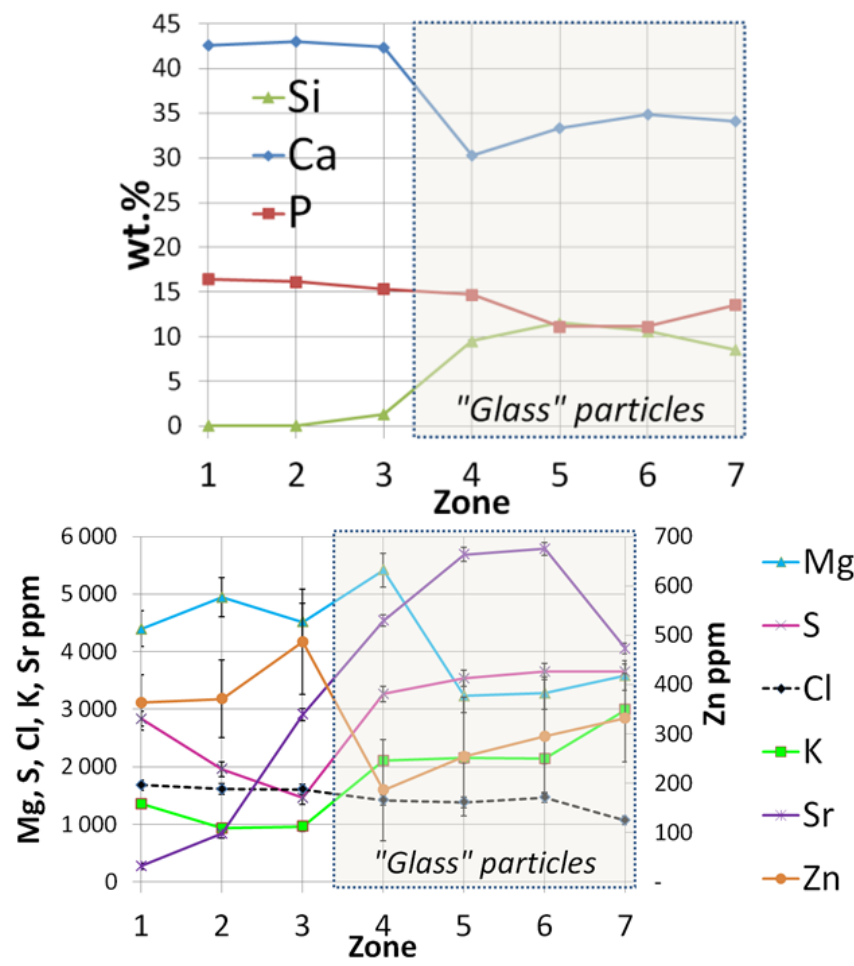

Figure 2: Concentration profiles along the B75Sr5/bone interface following the arrow indicated in Figure 1c. 


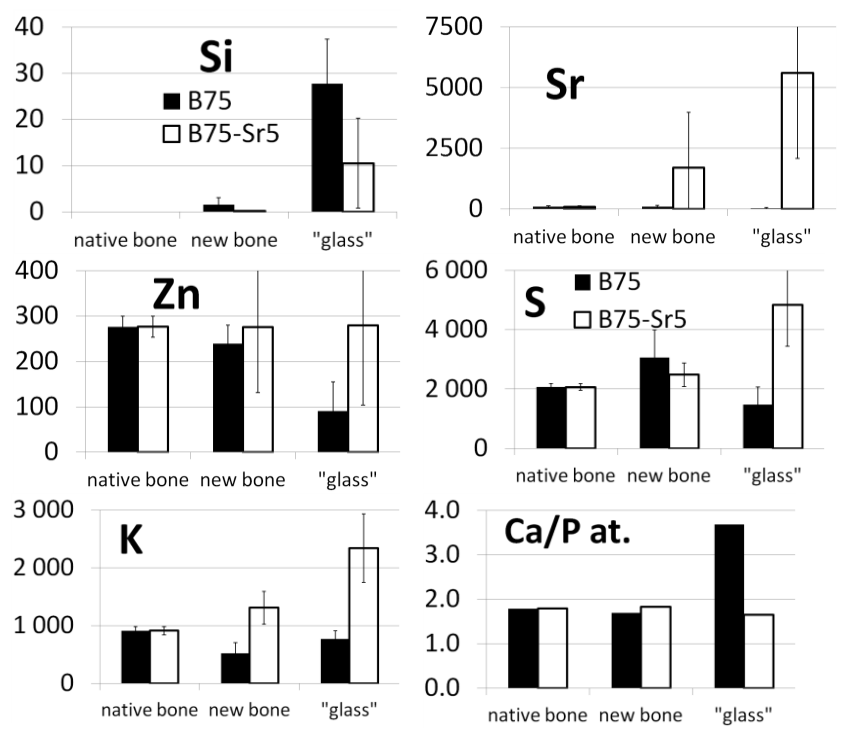

Figure 3: Composition in inorganic elements in native bone, new bone and inside the glass particles for B75 and B75-Sr5. Concentrations are given in wt.\% for Si and in ppm for Sr, Zn, S, K. The "error" bars correspond to standard deviation.

3 shows that Sr has been efficiently released from the glass. Part of the released $\mathrm{Sr}$ ions have been incorporated into the bone tissues in contact: indeed significant amounts of $\mathrm{Sr}$ are detected in the tissues up to several ten microns from the glass particles, a mean concentration being calculated to $1714 \mathrm{ppm} \mathrm{Sr}$ in new bone tissues. Interestingly, the contents in other trace elements also significantly differ depending on the type of glass. S, K and $\mathrm{Zn}$ concentrations are higher inside B75Sr5 particles. $S$ is implied in collagen synthesis, while $\mathrm{K}$ is an abundant cation found inside cells. $\mathrm{Zn}$ is recognized as a co- enzymatic factor and is an essential component of a large number of enzymes; the $\mathrm{Zn}$ content in bone tissues has been previously used as an indicator of the quality of bone formation [10]. It is thus especially meaningful here that $\mathrm{Zn}$ amount is significantly higher inside B75-Sr5 particles, reaching a mean value of $280 \mathrm{ppm}$ very close to the content in new and native bone.

\section{Conclusions}

Qualitative observations of bone formation through histological studies can be given more sense when coupled to a complementary quantitative microanalysis technique. Here the $\mu$ - PIXE analysis of implanted bioactive glasses gave important highlights on biomineralization in the presence of Sr. The high sensitivity of the technique allowed the detection of inorganic trace elements and indicated higher quality and advanced formation of bone mineral for $\mathrm{Sr}$ - doped glasses, compared to $\mathrm{Sr}$-free glasses. This is to be correlated with the delivery of Sr up to several ten microns around the implanted Sr-doped particles. The $\mu$-PIXE demonstration of the bioavailability $\mathrm{Sr}$ and their effects in vivo suggests Sr-doped bioactive glasses should be favorably considered for enhanced bone regeneration.

\section{Acknowledgment}

The Conseil Régional d'Auvergne and ANR (project "NANOSHAP" ANR-09BLAN- 0120) are acknowledged for financial support.

\section{References}

1. Buehler J, Chappuis P, Saffar JL, Tsouderos Y, Vignery A (2001) Strontium ranelate inhibits bone resorption while maintaining bone formation in alveolar bone in monkeys (Macaca fascicularis). Bone 29: 176-179.

2. Andersen $\mathrm{OZ}$, Offermanns $\mathrm{V}$, Sillassen $\mathrm{M}$, Almtoft $\mathrm{KP}$, Andersen $\mathrm{IH}$, et al. (2013) Accelerated bone ingrowth by local delivery of strontium from surface functionalized titanium implants. Biomaterials 34: 5883-5890.

3. Li Y, Li Q, Zhu S, Luo E, Li J, et al. (2010) The effect of strontium-substituted hydroxyapatite coating on implant fixation in ovariectomized rats. Biomaterials 31: 9006-9014.

4. Park JW (2011) Increased bone apposition on a titanium oxide surface incorporating phosphate and strontium. Clin Oral Implants Res 22: 230-234.

5. Wohl GR, Chettle DR, Pejović-Milić A, Druchok C, Webber CE, et al. (2013) Accumulation of bone strontium measured by in vivo XRF in rats supplemented with strontium citrate and strontium ranelate. Bone 52: 63-69.

6. Zheng Y, Jin W, Wang C, Yang M, Shen H, et al. (2009) The effects of strontium ranelate treatment on ovariectomized Sprague-Dawley rat tibia. NIMB 267: 2128-2131.

7. GorustovichAA, Steimetz T, CabriniRL, PortoLópezJM(2010)Osteoconductivity of strontium-doped bioactive glass particles: a histomorphometric study in rats. J Biomed Mater Res A 92: 232-237.

8. Lao J, Nedelec JM, Jallot E (2009) New strontium-based bioactive glasses: physicochemical reactivity and delivering capability of biologically active dissolution products. J Mat Chem 19: 2940-2949.

9. Isaac J, Nohra J, Lao J, Jallot E, Nedelec JM, et al. (2011) Effects of strontiumdoped bioactive glass on the differentiation of cultured osteogenic cells. Eur Cell Mater 21: 130-143.

10. Barbotteau Y, Irigaray JL, Moretto P (2004) PIXE characterization of tissues surrounding metallic prostheses coated with biological glasses. NIMB 215: 214-222. 